\title{
Quantification method of suspended solids in micromodel using image analysis
}

\author{
Ahmad Fadhil Jahari ${ }^{1}$ (D) Siti Rohaida Mohd Shafian ${ }^{2} \cdot$ Hazlina Husin $^{1} \cdot$ Norzafirah Razali $^{2} \cdot$ Sonny Irawan $^{3}$
}

Received: 21 September 2020 / Accepted: 24 March 2021 / Published online: 12 April 2021

(c) The Author(s) 2021

\begin{abstract}
Micromodel can provide valuable information to improve understanding of pore-scale transport phenomenon and can also be utilized to simulate the transport process at pore scale. This research aims to propose settlement option for quantification of suspended solids in micromodel. The micromodel is used to mimic the formation damage which occurs in reservoir formation that could simultaneously affect enhanced oil recovery. This is done by utilizing visual image interpretation through image analysis on micromodel chip. Following the quantification of suspended solids, the micromodel was injected with brine that eventually forms agglomeration. Images are taken from NIS-Element AR microscope automatically in RGB color profile and then made into grayscale and finally into binary modes. Since the micromodel is simulated in 2D form structure, the quantification method complemented with image analysis is focusing on the quantified area, $\mu^{2}{ }^{2}$ region of interest categorized into 3 main groups of area B05, M45 and T50, respectively. This research will explore on segmentation and thresholding processes of the visual data acquired from micromodel experiment. An image-based computational algorithm is programmed in MATLAB Image Processing Toolbox and ImageJ; hence, suspended solids in porous media could be quantified from the visual image executed in micromodel.
\end{abstract}

Keywords Image analysis $\cdot$ Micromodel $\cdot$ Suspended solids $\cdot$ Formation damage

\section{Introduction}

Oil and gas industry comprises of three major components which are upstream, midstream and downstream sectors. Petroleum production system (PPS) is one of the important parts constituted within upstream performance ( $\mathrm{Su}$ et al. 2018). PPS exposure is crucial to acquire better understanding on the fluid flow fundamentals along production system, likely to optimize well/ reservoir productivity and assessing performance of particularly well/ reservoir (Craig et al. 2018). For a single well system, primary elements constitute for PPS, reservoir, wellbore, tubular and related equipment and artificial lift equipment (Barbosa et al. 2019).

Ahmad Fadhil Jahari

ahmad_18001738@utp.edu

1 Petroleum Engineering Department, Universiti Teknologi PETRONAS, Tronoh, Perak, Malaysia

2 Hydrocarbon Recovery Technology PETRONAS Research Sdn Bhd, Kajang, Selangor, Malaysia

3 Petroleum Engineering Department, Nazarbayev University, Nur-Sultan, Kazakhstan
Hydrocarbon constituents habitually located in the wellbore which is contained within reservoir, from wellbore to wellbore subjected to tubular equipment providing path flow for fluid to flow upward by means of artificial lift; eventually, separation takes place to separate oil, gas and water. Meanwhile, PPS has never run way from various problems, for examples, deposition of wax and asphaltenes, fine movement and plugging, corrosion due to $\mathrm{CO}_{2}$ and $\mathrm{H}_{2} \mathrm{~S}$, heavy metals and radioactive materials, sand production and formation damage (Zhang et al. 2019a, b). Emphasizing here on the formation damage problem has caused lots of aftermath effect. Formation damage refers to the reservoir impairment due to such activities like drilling, completion, production, injection, stimulation or well invention during any interval of well which eventually results in significant reduction in well productivity (Yuan and Wood 2018).

Meanwhile, according to the previous studies conducted by a group of researchers from PETRONAS Research Sdn. Bhd. (PRSB), nano-silica has been used as chemical flood to mitigate fines migration (Rohaida et al. 2019). According to the result, application of nano-silica has shown good improvement in avoiding continuous migration of dispersed 
particles. Hence, the interest to further studies on this nanoparticle has been extended into pore-scale visualization by using micromodel to understand more on this formation damage (fines migration) mechanism in 2D porous media. As extension from previous research by PRSB, the micromodel initially is flooded with/ without nano-silica to observe the attachment of fines migration onto pore walls after injecting $2.5 \% \mathrm{NaCI}$.

Since at the beginning, this research keens to focus particularly on fines migration, as one type of formation damage. Nevertheless, during the preliminary stage of micromodel experiment another type of formation damage, mutual precipitation of soluble salt, was discovered. This refers to any precipitation generated from soluble salts, whether from the use of salt mud systems or from formation water (Zhang et al. 2019a, b). The precipitation was discovered when $2.5 \%$ $\mathrm{NaCI}$ injected into micromodel (base case). Since the only fluid introduced into the system is $\mathrm{NaCI}$, an assumption is made such that soluble salt precipitation might occur. Nevertheless, it is more unanimous to view the damage as generation of suspended solids adheres onto pore walls. The concern rises where suspended solids could be generated when brine is introduced. Formation water flow and water evaporation during brine injection can expedite formation of suspended solids (salt precipitates) with excessive loss of formation porosity and permeability, as correlated with reservoir physical properties. Since then, this research's interest pays thorough attention to this damage. Hence, this research study is focusing on developing a method to quantify area of suspended solids in micromodel by using image analysis. If left untreated, the worst case can be blocked wells leading to permeability impairment. Since this is highly unwanted, focus on this study is crucial.

As to visualize suspended solids retention and attachment on the grain surfaces of porous media, micromodel is introduced. Micromodel, as pore-level visualization study, offers both quantification and qualification studies through 2D porous media ( $\mathrm{Yu}$ et al. 2019). Pore-scale information is important for better understanding on the displacement process, imbibition and drainage. By utilizing this method, it promotes an enhancement to crucially study on the agglomeration mechanisms that may take place in a porous media. Instead, for further data analysis and interpretation on the images output executed from micromodel, comprehensive study through image analysis is complemented for this purpose. Suspended solid in reservoir is among formation damage that could lead to solid particles plugging and bridging within the pore throat of the well formation which then impedes to the fluid flow. Micromodel is utilized to visualize this phenomenon and analyzed thoroughly by image analysis method.

Several quantification research on micromodel embedded with image analysis have been conducted previously.
Studies conducted by researchers from Memorial University of Newfoundland, Canada, reported that implementation of micromodel has provided a good opportunity to explore on the multiphase fluid flow within the pore channel and fluid transport mechanisms (Mahmoodi et al. 2018). The variable of interest such as fluid saturation and other morphological features is quantified through a visual outcome executed from micromodel experiment which is then subject to further image processing/ analysis studies available in LabVIEW. Furthermore, expertise from China University of Mining \& Technology, China, has also extended the scope studies on micromodel embedded with image analysis performance by focusing on the impacts of pore-scale characteristics on the coupled supercritical $\mathrm{CO}_{2}$ dissolution and water flow in micromodel channel (Chang, Zhou, Kneafsey, Oostrom, \& $\mathrm{Ju}, 2019$ ). An image processing approach, ImageJ, is introduced to visualize and quantify the dynamic dissolution process in micromodel. Another study published by Journal of Petroleum Science and Engineering, focusing on the pore-scale swelling behavior of clays in pore channel, has concluded that the effective porosity in micromodel during low salinity water (LSW) flooding effectively can be quantified through comprehensive image processing, MATLAB Image Processing Toolbox (Sharifipour et al. 2019). Thus, this research will explore on severity of damage thickness in 2D micromodel executed from extensive image analysis to fill the research gaps, as part of novelty.

\section{Methodology}

Comprehensive study through image analysis is conducted for the purpose of data analysis and interpretation on the executed images. Image analysis is a computer-based process of recognizing attributes (image recognition) and extracting quantitative information from images (Mahmoodi et al. 2018). It is a morphometric technique used to acquire quantitative data from digital images using analysis software that segments pixels in a digital image based on color or intensity (RGB, grayscale). The process commences with the input of an image and over with the output of numerical data (Khorshidian et al. 2018). Simply, it means input image is modified by one or more mathematical algorithms to generate output image that is enhanced in several way prior further analysis.

There are many different techniques used in automatically analyzing images. Examples of image analysis techniques include: geometric operations, neighborhood and block operations, linear filtering and filter design, transform, image analysis and enhancement, binary image operations and region of interest operations (Yang 2019). Further data analysis and interpretation on the micromodel output image will utilize these 3 methods from overall image analysis: image 
analysis and enhancement, binary image (BW) operations and region of interest (ROI) operations. Firstly, image analysis and enhancement comprise of two important processes which is segmentation and thresholding. Segmentation is the first step in image analysis, a process of partitioning a digital image into multiple segments (sets of pixels) and clustering pixels into salient image (Shen and Maa 2016). Image segmentation techniques can be divided into three groups which are edge based, region based and pixel based. Focus on pixel based, having thresholding and clustering elements. Another important process is thresholding, run after segmentation process completed. Thresholding is a technique for partitioning image directly into region based on intensity value and property of these value (Liu et al. 2017).

Instead, the image analysis process is highly dependent on the color profile which needs the image conversion from RGB to grayscale and then binary image (BW). After the first method (image analysis and enhancement), then move to binary image (BW) operation. For further reference on the color profile conversion (RGB-grayscale-binary), RGB stands for Red, Green, Blue. Each color pixel is made up of these 3 colors (Xiao et al. 2018). The intensity of this color profile is from 0 to 255; for example, pure red pixel is $(255,0,0)$. Grayscale intensity contains 256 colors $(0-255)$, and it is a combination of RGB intensity in a specified ratio. 0 indicates black, 1 until 254 represent gray intensity and 255 is white (Belati and Cajaiba 2018). Binary image contains only black (0) and white (1) colors. For grayscale change to binary, grayscale intensity profile $0-127$ turns to black (1) while 128-255 would switch to white (1), simultaneously (Hernández et al. 2018). Finally, the 3rd method (region of interest operation) from image analysis is conducted to quantify the image accordingly from the BW output. Figure 1 indicates the flow chart for the process and assumption made for further data analysis interpretation.
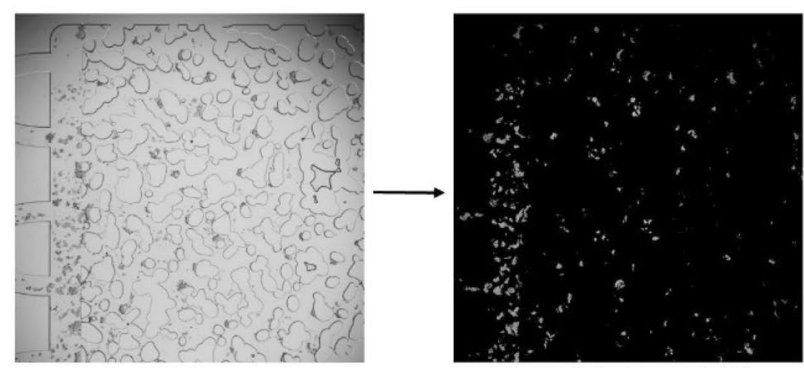

RGB Image

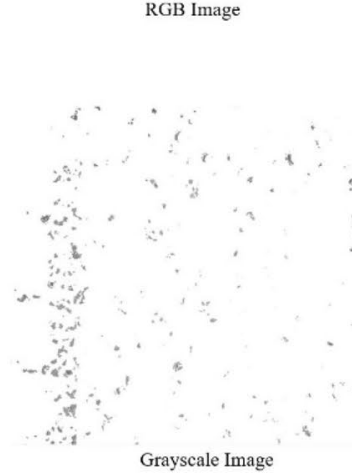

Grayscale Image

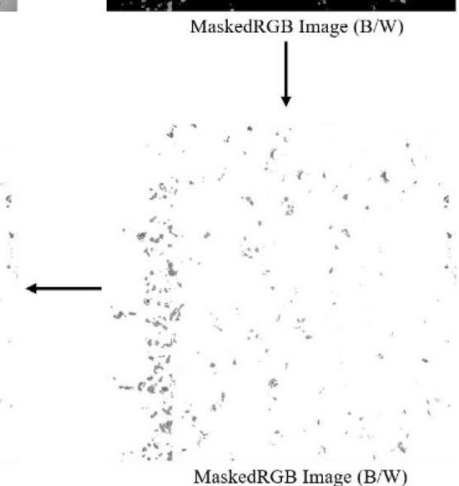

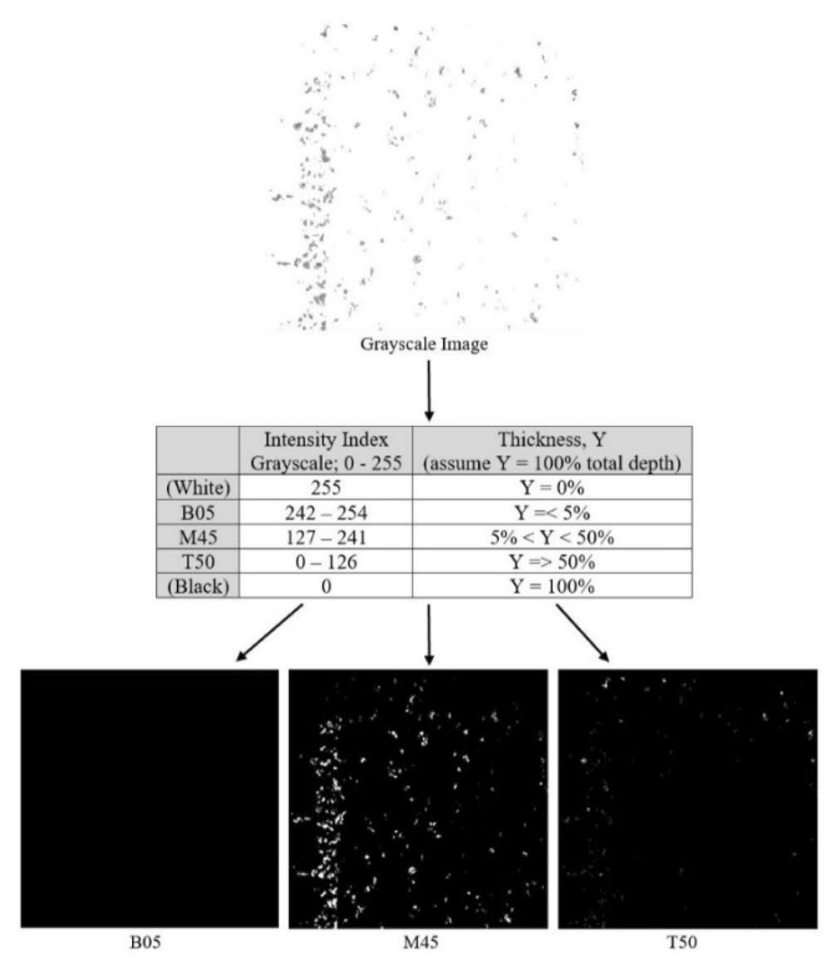

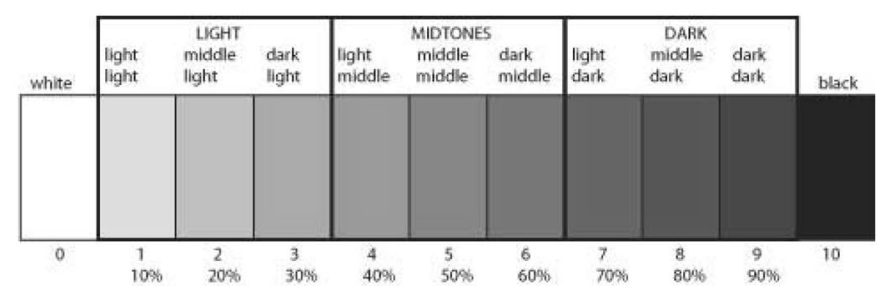

Fig. 1 Process flow chart and assumption 
Fig. 26 regions of interest (ROI) of a micromodel

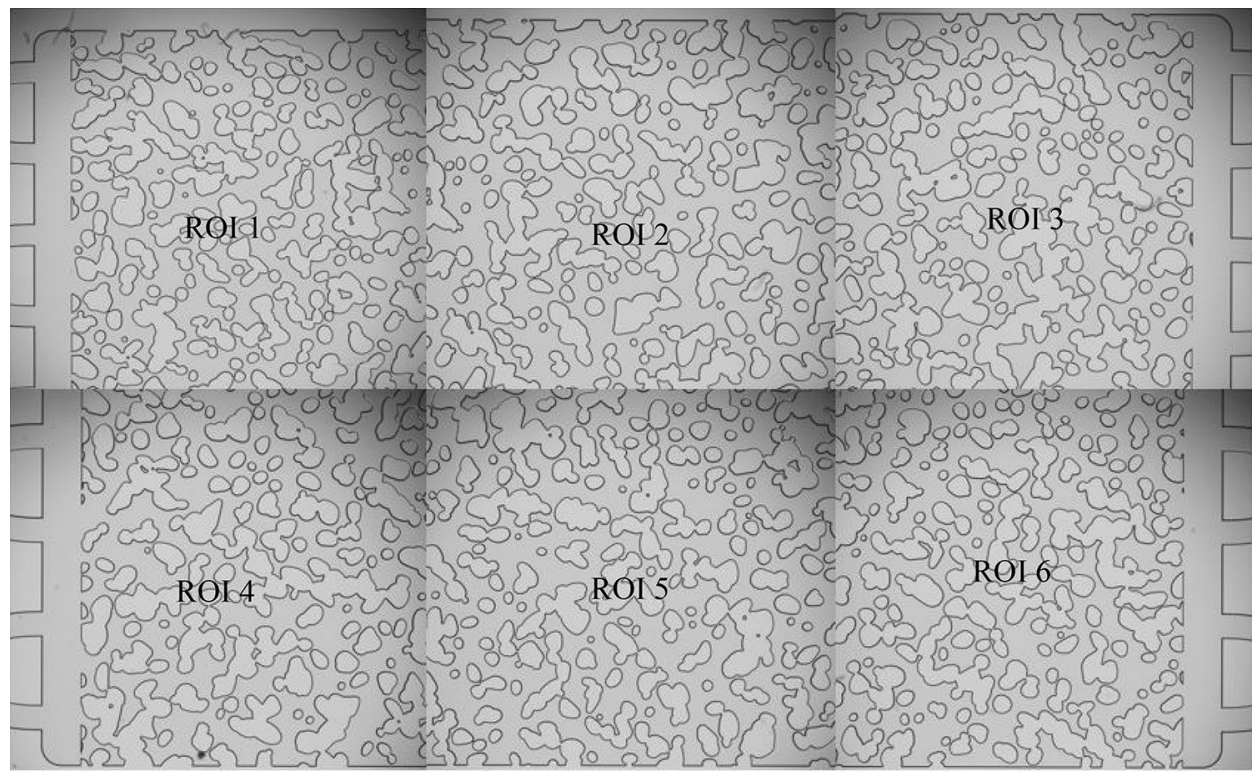

\section{Materials}

Chemical used for preparing synthetic brine (formation water) is $2.5 \%$ of $\mathrm{NaCI}$ from Sigma-Aldrich. Brine was injected with a syringe pump (Chemyx Stailess Stell Syringe Fusion) while pressure difference was recorded through pressure transducer (Nexus 3000 Chemyx Inc.).

The main part of micromodel setup is the micromodel chip fabricated from glass which consists of microfluidic channels and pore network. Micromodel chip was retrieved from Micronit, with topological structure defined as physical rock. Total chip dimension was $45 \times 15 \mathrm{~mm}$, with microfluidic network region of $20 \times 10 \mathrm{~mm}$ (Fig. 2).

Connected to micromodel chip is the microscope. NISElements AR microscope was used for imaging the suspended solids; then, the captured images were recorded in computer control image analysis (HP Z440 Xeon) and further interpretation through image analysis.

Further data analysis and interpretation on executed images were done utilizing processing toolbox, MATLAB Image Processing Toolbox and ImageJ. These tools are used for the image analysis purpose (Fig. 3).

\section{Characterization of micromodel}

Six regions of interest (ROI) have been identified at inlet, middle and outlet site in a newly micromodel. These specified ROIs were used for further studies. The micromodel is further characterized on their physical properties, pore $\&$ grain size and porosity. The images are taken by using NIS-Element AR microscope at $2 \times$ resolution.

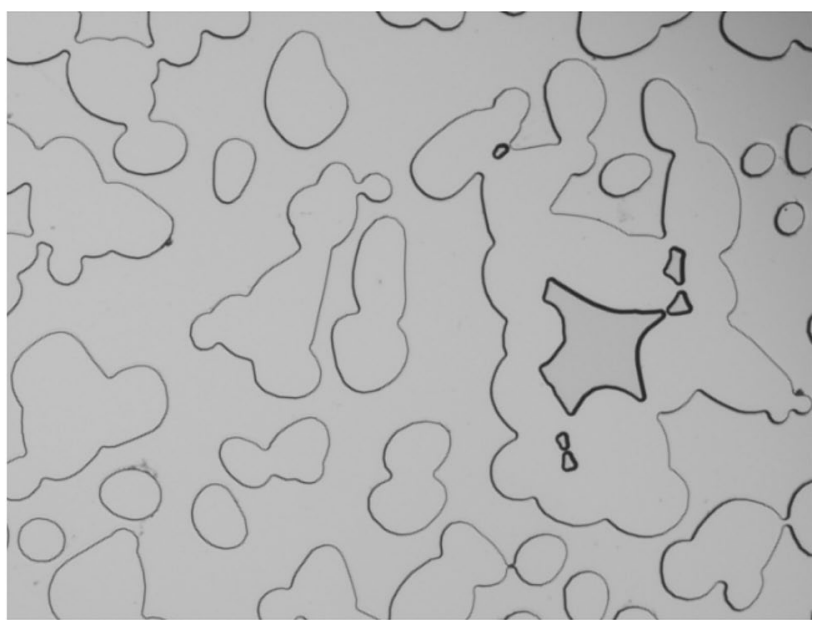

Fig. 3 A section of micromodel representing physical rocks

Table 1 Micromodel: pore and grain size

\begin{tabular}{llll}
\hline & Fine & Medium & Coarse \\
\hline Pore size $(\mu \mathrm{m})$ & $36.33-87.76$ & $90.31-191.4$ & $205.37-441.46$ \\
Grain size $(\mu \mathrm{m})$ & $110.27-186.08$ & $209.82-305.02$ & $387.13-705.9$ \\
\hline
\end{tabular}

\section{Micromodel: pore and grain size}

The pore and grain size of micromodel is summarized in three groups, categorized as fine, medium and coarse as referred in Table 1. These data have been determined by using Annotations \& Measurements tool on NIS Element AR microscope software-embedded computer control 
image analysis. The pore and grain size output indicates the overall ROIs studies.

\section{Micromodel: porosity}

Further characterization is implemented to determine the porosity for each ROI. This is done by using computational method utilize MATLAB and ImageJ software. Initially, captured image is in RGB and needs further image conversion into grayscale then binary image (RGB $\longrightarrow$ grayscale $\longrightarrow$ binary image) which is done in ImageJ for this conversion purpose. In ImageJ, the RGB image is converted into RGB stack type which literally makes the image become grayscale that has only black to white intensity color $(0-255)$. Next, stack to binary conversion using Huang method that give black and white image ( 0 and 1$)$. The output from intensity color range more to dark and light area would shift to black (0) and white (1) simultaneously. Wand tool is selected to fill the grain space with white color while black color denotes to pore space; then, the overall binary image is inverted which gives grain and pore space into black and white eventually. The sequence of the conversion process is referred in Fig. 4.

Binary image can provide information on available pore space, well connectivity and flow path. The output in Fig. 4 (binary image) is then transferred into MATLAB for porosity determination purpose. Height and width of the binary image are input in MATLAB to give overall area. Then, MATLAB algorithms are generated to execute the black and white space area. Hence, calculated porosity is displayed and determined. The MATLAB algorithms used for this purpose are imread, bwmorph, imshow, sum and sprintf to read image, morphology purpose, display image, sum up for black/white area and display calculated porosity. The porosity for each ROI is referred in Table 2.

\section{Micromodel: brine injection}

$40 \mathrm{ml}$ of $2.5 \% \mathrm{NaCl}$ is injected at $0.1 \mathrm{ml} / \mathrm{min}$ into the micromodel. Shortly thereafter, formation of suspended solids is identified and can be seen attached on the grain surface. The executed images are captured at specified ROIs and are referred in Fig. 5.

\section{Results and discussion}

\section{Segmentation process of suspended solids}

The captured image from NIS-Element AR microscope is input into MATLAB Image Processing Toolbox. Then, RGB image is thresholded using code from the colorThresholder App that finally exhibits createMask (RGB) form. The color space and minimum/ maximum values for each channel of the color space were set in the App and give result in a binary mask BW and a composite image maskedRGBImage. Figure 6 shows the sequence process. Initially, RGB image is converted to chosen color space, and then, define the thresholds ( $\mathrm{min} / \max$ ) based on histogram settings. Next, mask is created based on chosen histogram thresholds that finally exhibit output. Shortly thereafter, invert mask is applied. The output maskedRGBimage been initialized based on input image and background pixels is set results as in Fig. 6. This process (invert mask) will make the RGB image and BW on maskedRGBimage to emerge as BW image and RGB on InvertMask-image (Table 3).

According to Fig. 7, BW images indicate agglomerate suspended solids have been segmented using Color Thresholder HSV method. The minimum/ maximum values for each channel of the color space are defined based on histogram settings and result in a binary mask BW with composite image maskedRGBImage. These findings denote that optimization of segmentation process is important in order to execute suspended solids from the input image. As the image analysis particularly on segmentation process is complete, prolonged analysis will be initialized on thresholding process.

\section{Thresholding process of suspended solids}

\section{1st Thresholding Process}

The 1st stage of thresholding process will be utilizing ImageJ for image processing purpose. The 'maskedRGBImage' from segmentation process is input into ImageJ; then, the image is adjusted for Color Threshold. On the Threshold Color folder, the information filled for the thresholding method, threshold color and color space are default, white and HSB (hue, saturation, brightness). Applying this will directly invert binary mask BW from black to white while maintaining the segmented region in RGB image, as shown in Fig. 8. The purpose of this process is to enhance the visualization and emergence of suspended solids in their ROIs. This is achieved by applying multiband thresholding. This is typically done in order to separate "object" or foreground pixels from background pixels to aid in image processing. Color images can also be threshold. One approach is to designate a separate threshold for each of the RGB components of the image and then combine them with an AND operation. Thus, the HSL, HSV and HSB color models are more often used (Pham et al. 2007).

\section{2nd Thresholding Process}

The 2nd stage of thresholding process involves conversion of image from RGB to grayscale to binary, as referred in

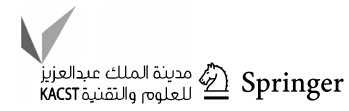


Fig. 4 Sequence of image conversion from (i) RGB image to (ii) grayscale image, and finally to (iii) Binary image

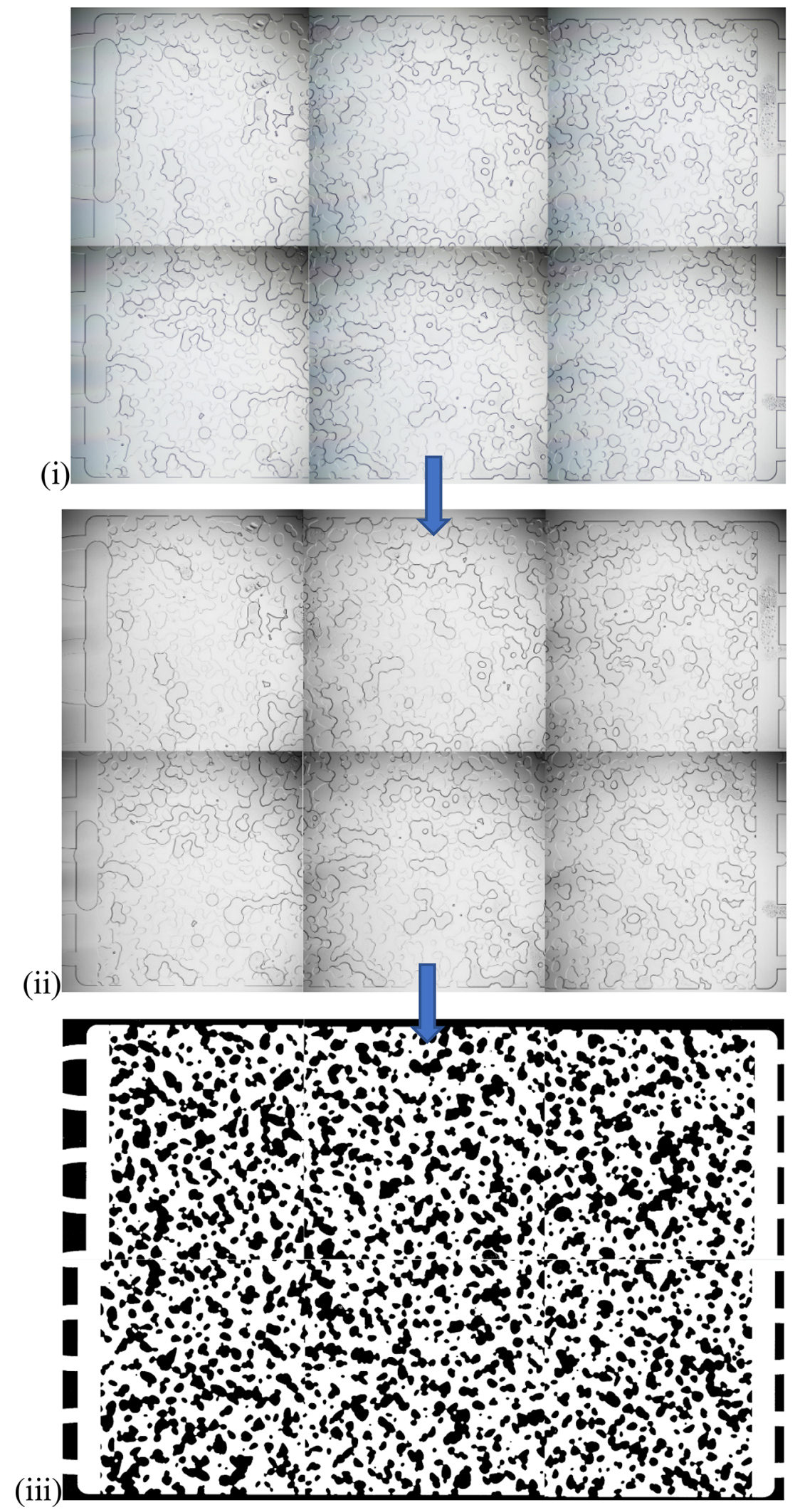


Table 2 Micromodel: porosity

\begin{tabular}{ll}
\hline ROI & Porosity \\
\hline ROI 1 & 0.5018 \\
ROI 2 & 0.5384 \\
ROI 3 & 0.5069 \\
ROI 4 & 0.5103 \\
ROI 5 & 0.533 \\
ROI 6 & 0.5165 \\
\hline
\end{tabular}

Fig. 9. This is done by using MATLAB Image Processing Toolbox using code rgb2gray and im $2 b w$. This step is crucial to provide validity on which image needed to prioritize for finalization stage which is quantification of suspended solids. During rgb2gray process (RGB to grayscale), merge of red, green, and blue color is converted into intensity range color from black to white. During im $2 b w$ process (grayscale to binary), black and white intensity color is converted to black and white color. Grayscale intensity below than 128 would shift to black and greater than 128 shifts to white. This conversion (im2bw) method brings significant reduction in suspended solids on the output binary image. From naked eye, estimated around $30-40 \%$ could diminish using this method. Hence, for further stage involving quantification of suspended solids will utilize rgb2gray method that gives grayscale image as output (0-255). As reference, 255 refers to white and 0-254 refers to solid intensity level.

The overall output of grayscale image is presented in Fig. 10.

\section{Quantification of suspended solids}

The quantification process of suspended solids commenced after thorough image analysis; segmentation and thresholding process is complete. This is done by using MATLAB Image Processing Toolbox. The suspended solids are quantified in the form of area $\left(\mu \mathrm{m}^{2}\right)$ invaded in the specified ROIs. In order to quantify the area of invaded region, the input grayscale image is used for this purpose. Acknowledge that grayscale image would come in the form of intensity black to white color, from 0 to 255 , where $0=$ black, 1 to $254=$ black to white intensity level and $255=$ white, overall, with 256 $\mathrm{BW}$ color (uint8 bit $=256$ ). The grayscale image is further analyzed accordingly by using their grayscale index (0-255).

Furthermore, there are 3 types of groups used defined as T50, M45 and B05 for quantification purpose. As reference, T50 indicates to intensity color from dark gray to medium light (0 until 126), M45 denotes to intensity color from medium to light ray (127 until 241) and B05 refers to intensity color from light to very light gray (242 until 254). Since the micromodel is in 2D figure, the depth is unknown, let be

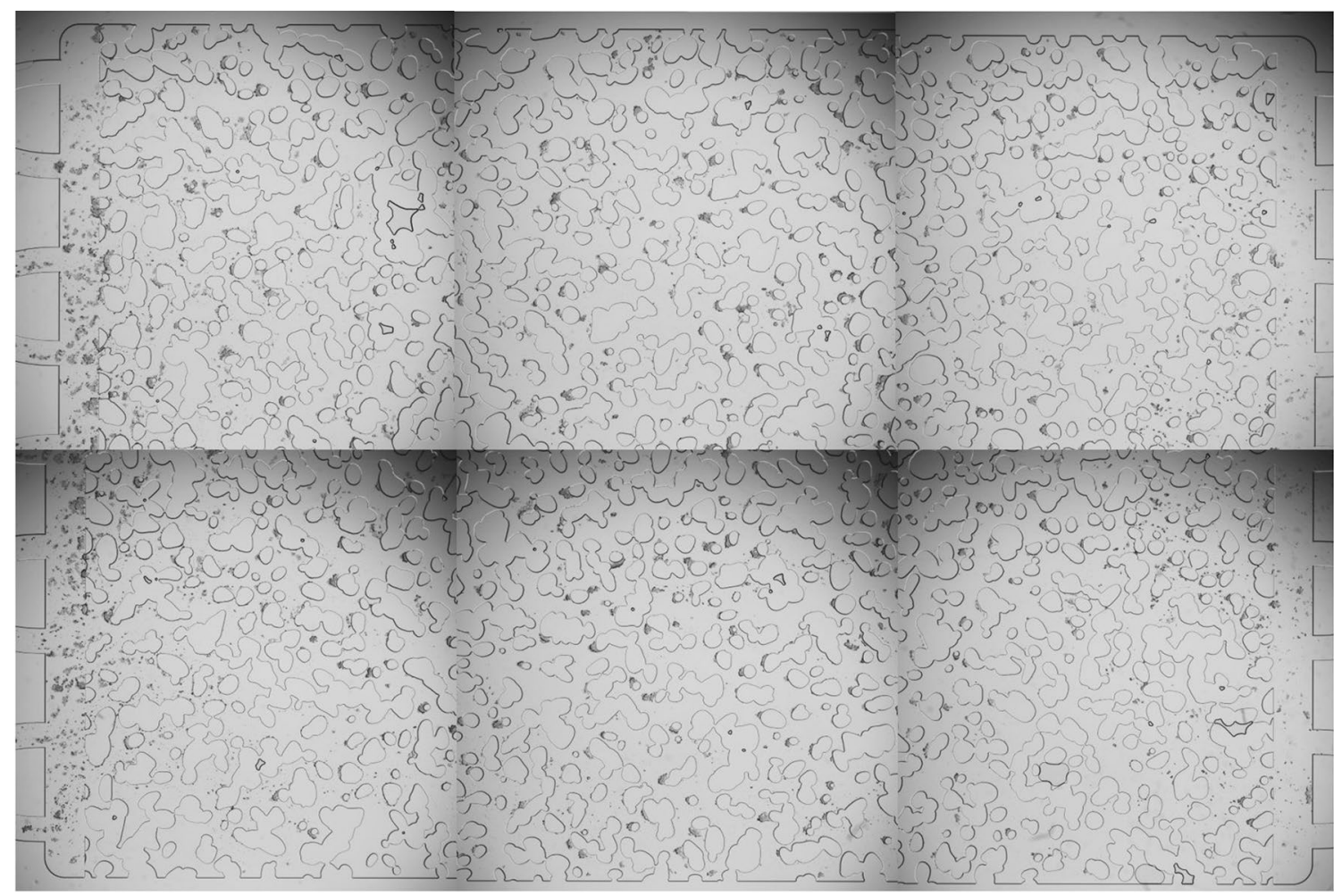

Fig. 5 Brine injection 
Captured image from NIS-Element

AR Microscope
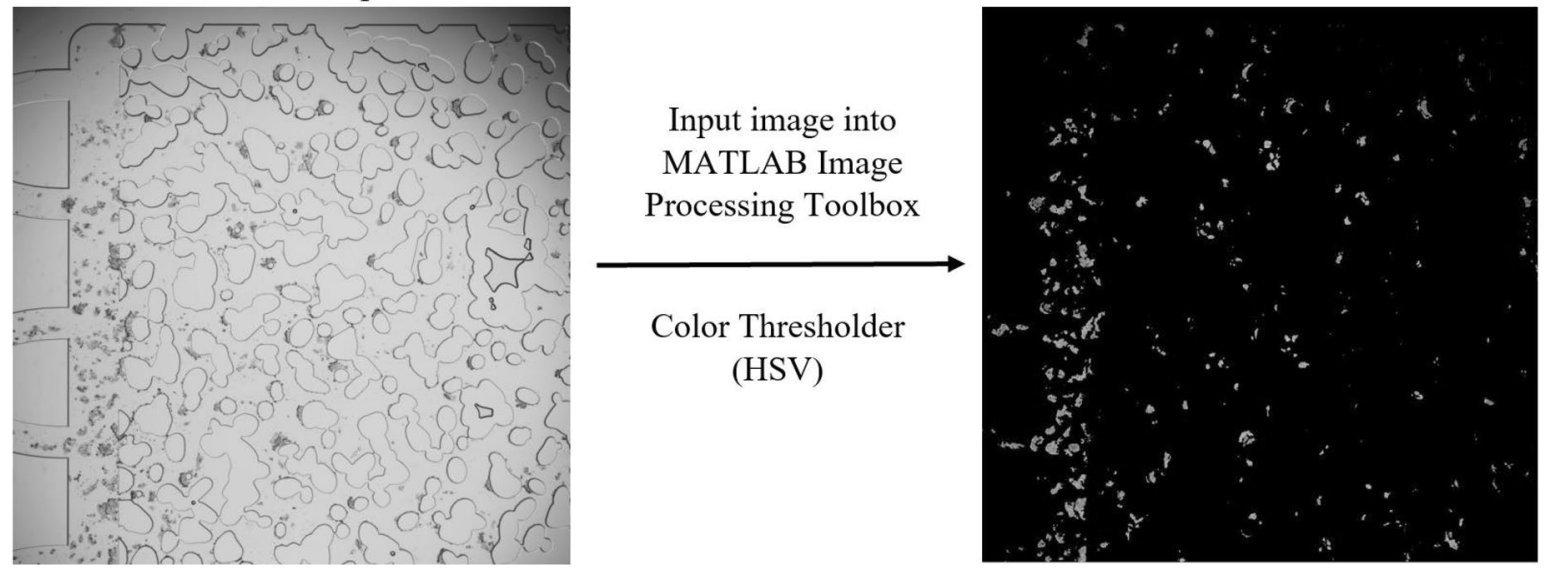

Fig. 6 1st segmentation process

the depth $=\mathrm{Y}$ (assume $\mathrm{Y}=100 \%$ total depth). For detail, as the intensity index given is 0 (black), it means the whole $2 \mathrm{D}$ space is invaded by salt precipitation, literally invaded depth, $\mathrm{Y}=100 \%$, while as if the intensity index is 255 (white), it refers none of the salt precipitation invades that region which means invaded depth, $\mathrm{Y}=0 \%$.

Group T50 indicates to part of suspended solids that will invade the 2D space (depth) beyond 50\% from actual micromodel depth, from 0 until 126 ( $0.5 \mathrm{Y}$ until $1 Y)$. For better understanding, having 126 indexes means that probably there is high feasibility that the suspended solids depth $=0.5 \mathrm{Y}$ while 0 index approximately $1 \mathrm{Y}$ has been invaded. Similarly, group M45 (0.05Y until 0.5Y) and B05 (0Y until 0.05Y) refer to part of suspended solids categorized in their own Y. The groups (T50, M45 and B05) refer to the severity of the invasion in 2D pore space. For example, having much T50 would likely lead to severe permeability impairment since the pore space depth could be blocked by suspended solids almost $>50 \%$ height from the overall depth.

Table 4 presents the images for each ROIs accordingly with their categorized group, T50, M45 and B05. Each grayscale image is executed into these 3 categorized groups; then, executed image will be inverted into BW image to exhibit the suspended solids and background images in white and black color. The given output (from T50, M45 and B05) is further discussed on their specified ROIs with respect to suspended solids invasion. As mentioned earlier, the micromodel has been finalized into 6 ROIs from the inlet, middle and outlet location. There are known as ROI1 (top-inlet), ROI2 (top-middle), ROI3 (top-outlet), ROI4 (bottom-inlet), ROI5 (bottom-middle) and ROI6 (bottom-outlet). For further reference, detailed properties of executed images from NISElement AR microscope having dimensions $1608 \times 1608$ pixels and in relation with SI unit are $3.67 \mu \mathrm{m} / \mathrm{px}$. Initially, area of image is in pixel ${ }^{2}$ and needs to convert into $\mu \mathrm{m}^{2}$ with given correlation conversion.

The first discussion will be focusing on the quantification area on the group T50 (dark gray to medium light, 0 until 126 intensity). As fluids enter the micromodel, they invade the top and bottom region, simultaneously. When looking at the invasion region on the top site from ROI1, ROI2 and ROI3 (inlet to outlet), the invasion area of T50 becomes less from $8.18 \times 10^{4}, 5.75 \times 10^{4}, 2.82 \times 10^{4} \mu \mathrm{m}^{2}$. The trend also shows the same declination of invasion area on bottom site from ROI4, ROI5 and ROI6 (inlet to outlet), $1.25 \times 10^{5}$, $4.69 \times 10^{4}, 3.99 \times 10^{4} \mu \mathrm{m}^{2}$. For both top and bottom regions, as suspended solids migrate from the inlet to outlet, approximately about $48.85 \%$ and $59.02 \%$ are likely to attach and adhere at the inlet ROIs (ROI1 and ROI4) while $16.83 \%$ and $18.85 \%$ would attach at the outlet ROIs (ROI3 and ROI6). Furthermore, according to the results, it indicates that much sedimentation occurred at the bottom site in comparison with top site. Suspended solids attached to the pore surfaces are highly related with attractive-repulsive forces applied on it. High sedimentation is discovered at the inlet since this location is prone to get attracted with suspended solids, at first invasion. Over time, attractive forces present at front grains are not adequate to provide firm attachment on the surface, yet part of suspended solids could adhere on it while the remnant would naturally migrate to the other site available for attraction forces for them to adhere on. This phenomenon results in retention of suspended solids from one location to others as they migrate from inlet to outlet, respectively. Instead, due to the topological structure of micromodel physical rock seems high; likely fluids path flow tends to migrate at bottom region and then invades top side, resulting in high sedimentation discovered at bottom the most. 
Table 3 Binary image output of group T50, M45 and B05

\begin{tabular}{|c|c|c|c|c|}
\hline $\begin{array}{c}\text { Image } \\
\text { ID }\end{array}$ & Grayscale Image & $\begin{array}{c}\text { Group T50 } \\
\text { Color: Dark gray to } \\
\text { medium light } \\
\text { Intensity: } 0 \text { - } 126\end{array}$ & $\begin{array}{c}\text { Group M45 } \\
\text { Color: Medium to } \\
\text { light ray } \\
\text { Intensity: } 127 \text { - } \\
241\end{array}$ & $\begin{array}{c}\text { Group B05 } \\
\text { Color: Light to } \\
\text { very light gray } \\
\text { Intensity: } 242 \text { - } \\
254\end{array}$ \\
\hline ROI1 & 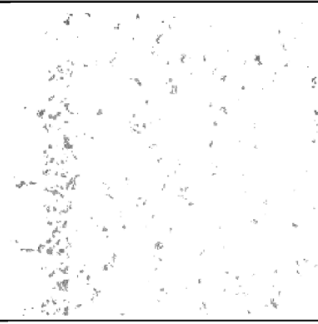 & 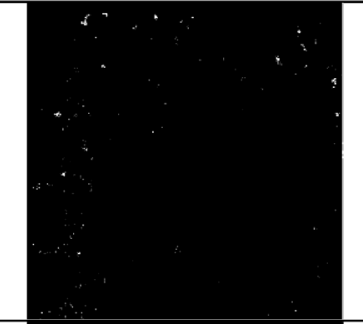 & 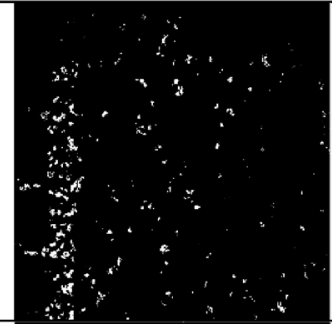 & \\
\hline 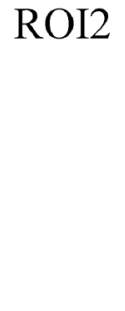 & 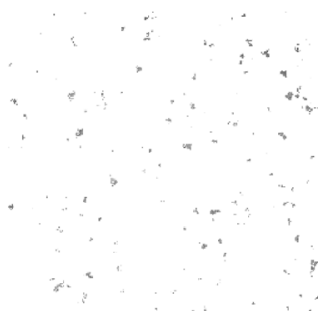 & : & 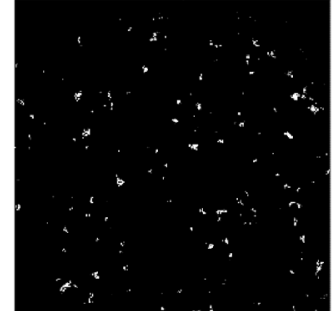 & \\
\hline ROI3 & $\begin{array}{l} \\
\vdots \\
\ddots\end{array}$ & $\ldots=$ & $\begin{array}{l} \\
\cdots\end{array}$ & \\
\hline ROI4 & 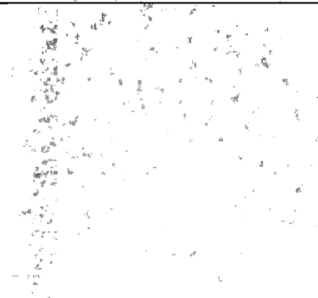 & $+\cdots$ & 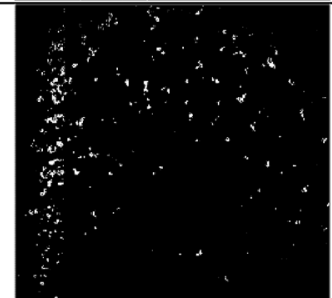 & \\
\hline ROI5 & 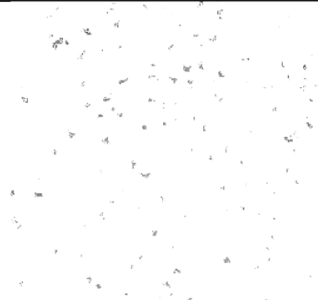 & $\cdots$ & 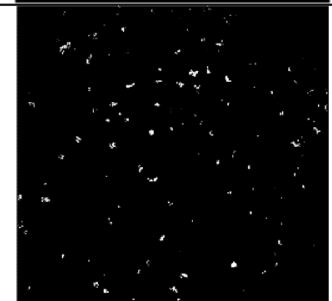 & \\
\hline ROI6 & , & . $:$ & $\begin{array}{lll}\cdots & \vdots \\
\ddots & \ddots & \ddots \\
\ddots & \ddots\end{array}$ & \\
\hline
\end{tabular}




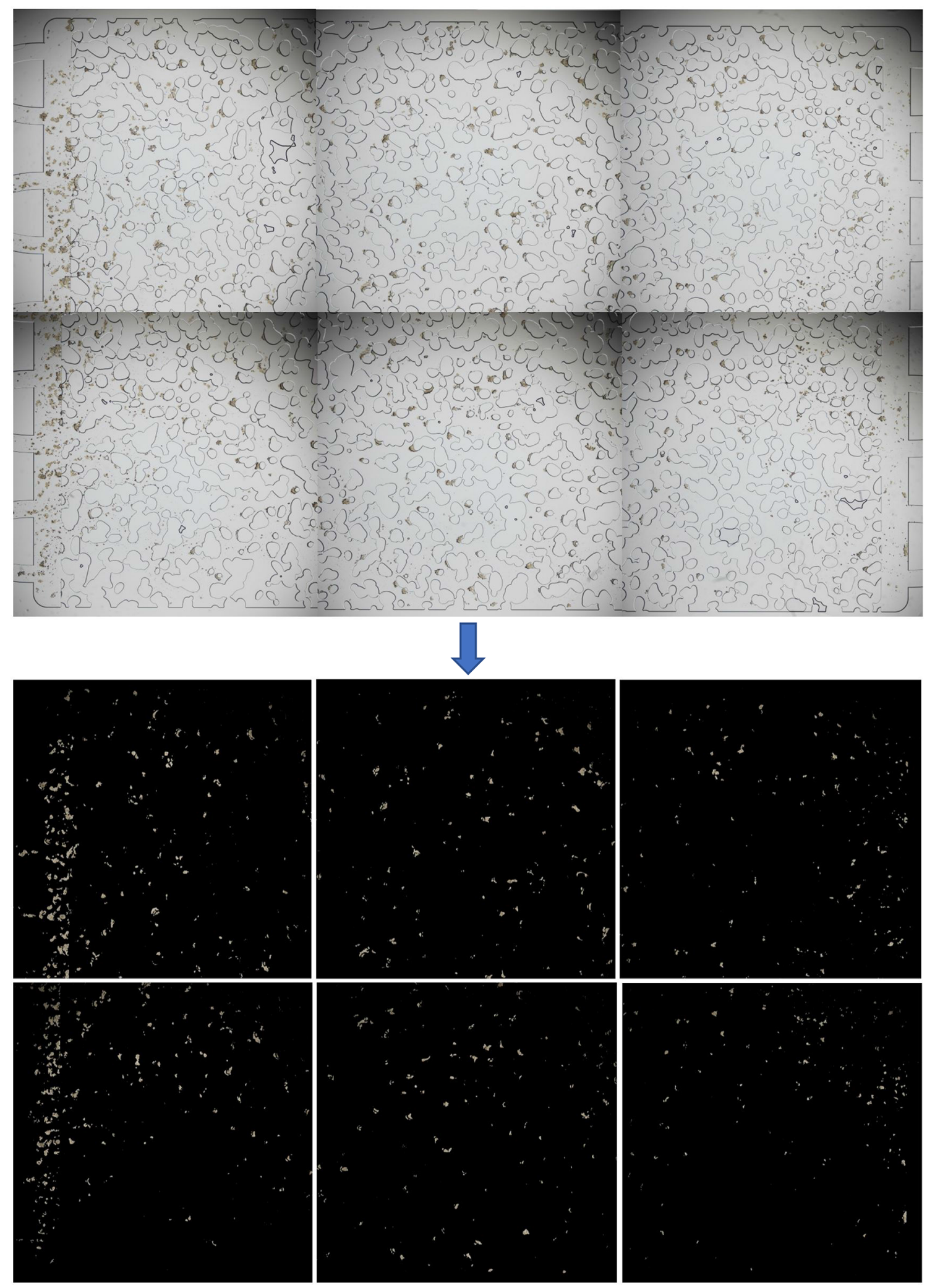

Fig. 7 1st segmentation 

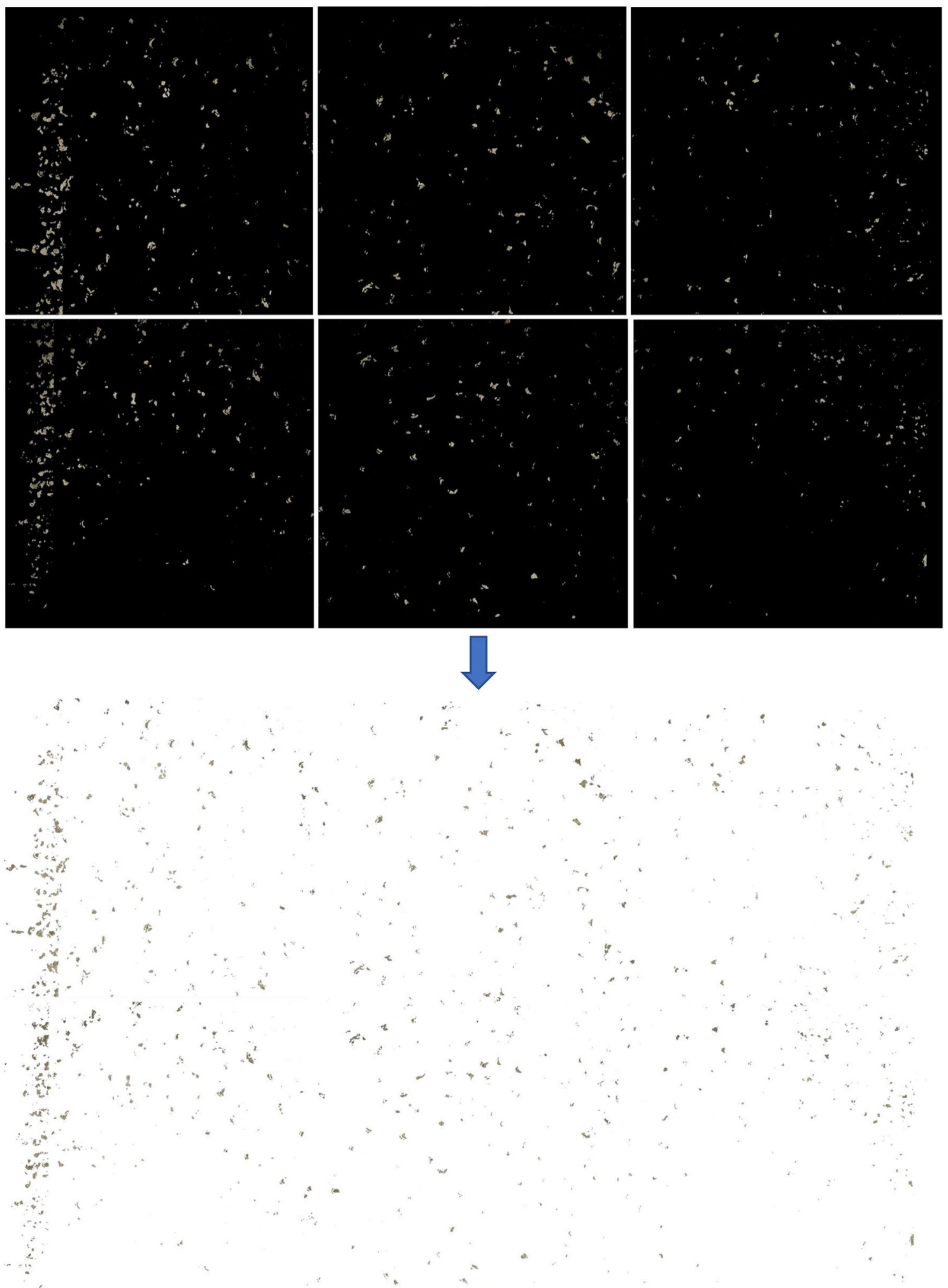

Fig. 8 1st thresholding process 
Fig. 9 2nd thresholding process (image conversion)

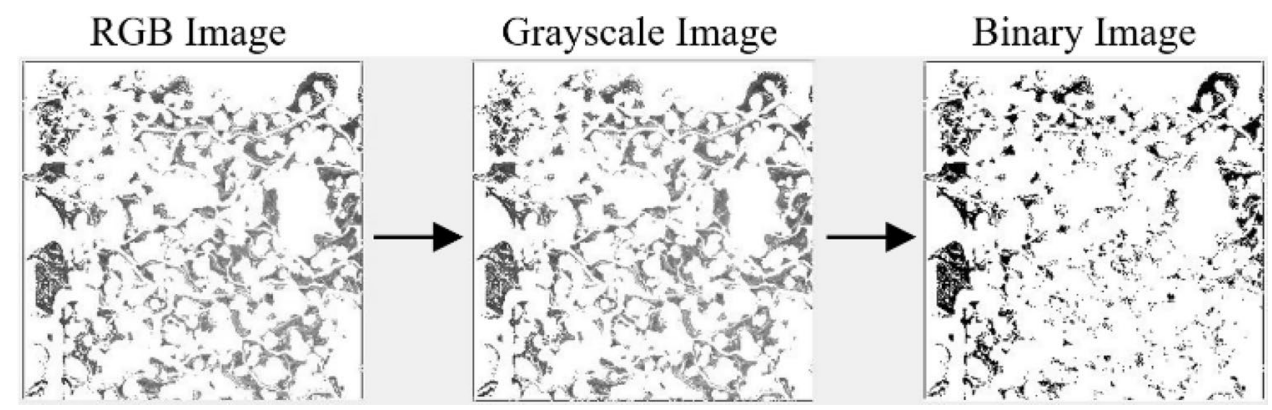

Fig. 10 rgb2gray process (grayscale image as output) 
Table 4 Quantification area of group T50, M45 and B05

\begin{tabular}{|c|c|c|c|c|c|c|}
\hline & \multicolumn{3}{|c|}{ Total area (in $\mathrm{px}^{2}$ ) @ reservoir resolution } & \multicolumn{3}{|c|}{ Total area (in $\mu \mathrm{m}^{2}$ ) @ reservoir resolution } \\
\hline & B05 & M45 & $\mathrm{T} 50$ & B05 & M45 & $\mathrm{T} 50$ \\
\hline ROI1 & 0 & $56,834.69$ & 6071.11 & 0 & $765,500.82$ & $81,771.18$ \\
\hline ROI2 & 0 & $27,033.53$ & 4266.44 & 0 & $364,111.89$ & $57,464.3$ \\
\hline ROI3 & 0 & $20,022.85$ & 2091.72 & 0 & $269,685.79$ & $28,173.13$ \\
\hline ROI4 & 0 & $39,115.86$ & 9277.62 & 0 & $526,847.57$ & $124,959.3$ \\
\hline ROI5 & 0 & $20,009.68$ & 3479.58 & 0 & $269,508.38$ & $46,866.17$ \\
\hline ROI6 & 0 & $14,325.43$ & 2962.62 & 0 & $192,947.8$ & $39,903.28$ \\
\hline
\end{tabular}

Notes: $3.67 \mu \mathrm{m} / \mathrm{px}$
On the other hand, the trend for group M45 does not show significant different from T50. Focusing on the topside region from ROI1, ROI2 and ROI3, the invasion area for M45 slightly declines from inlet to outlet, indicating $7.66 \times 10^{5}, 3.64 \times 10^{5}, 2.7 \times 10^{5} \mu \mathrm{m}^{2}$. Similarly, declination trend is also finalized at the bottom side of micromodel from the inlet to outlet (ROI4, ROI5 and ROI6) gives result of $5.27 \times 10^{5}, 2.7 \times 10^{5}, 1.93 \times 10^{5} \mu \mathrm{m}^{2}$. Furthermore, based on the result it indicates that there is no part of B05 sedimentation. Literally, mild thickness of suspended solid which is below than $5 \%$ is unlikely to occur.

From the overall output (T50 and M45), it indicates that by time suspended solids invaded the pore region, most likely the inlet part will experience high adherence of these particles and become less at the outlet part. Suspended solids remain attached to pore walls when there are enough electrostatic and van der Waals forces. The effects of the intermolecular forces on the stability of disperse systems can be described in terms of the forces between the colloidal particles determined by the summation of the intermolecular forces over many molecules (Paden 2016). Two main interacting forces in affecting colloid systems are van de Waals attractive forces and electrostatic repulsive forces. Attractive forces tend to destabilize colloids, whereas repulsive forces generally impart stability. van de Waals is originated from the dipole-dipole interactions, while their attractive force increases more and more rapidly as the molecule approach (Adrian Parsegian 2005). On the other hand, oppositely charged ions are preferentially attracted toward the surface, and ions of the same charge tend to be repelled away. The combination of the charged surface and the unequal distribution of cation and anion near the surface is called the electrical double layer (Moya 2015). As repulsive forces exceed attractive force, they detached from pore surfaces. As suspended solids enter the micromodel, the inlet part is exposed initially with them which literally causes attachment on grain walls. At time when the available attractive forces could not attract, much suspended solids adhere on their surfaces and hence will limit the attachment at front site and begin to migrate onwards. Instead, when compared accumulated suspended solids at both top and bottom regions in terms of invasion area of group T50 and M45, top side gains much invaded region of group M45 while T50 at bottom site. There is feasibility of pore restriction and channel that drive to this outcome since factors like available pore space, pore space connectivity and tortuosity index might affect simultaneously.

Besides that, by referring to Table 4 it indicates that high accumulation of suspended solids area under group M45 is discovered at all ROIs (ROI1 until ROI6) comparing with group T50 and B05. Output images prove that as seen M45 area is scattering throughout the space. In terms of scattering index, M45 possesses the highest scattering index followed by T50 and B05. All the output (quantification area of T50, M45 and B05) acquired is crucial for determination and thorough understanding of afterward permeability/porosity that has been severely affected and performance of chemical injection to cause solid attachment/detachment. In comparison for their severity of thickness in 2D micromodel (ROI1 until ROI6), sedimentation area for group B05 is negligible while M45 recorded the highest remark. Throughout 6 ROIs, if such sedimentation of suspended solids occurs, $80.83 \%-90.54 \%$ would fall under group M45 which means the severity of thickness in 2D micromodel is below or equal to $50 \%$ from the overall depth. Basically, further interpretation could be done to enhance the current $2 \mathrm{D}$ visualization into $3 \mathrm{D}$ by utilizing ImageJ, Interactive 3D Surface Plot. As referred in Fig. 11, ROI1 images are processed using ImageJ; subsequently, create 3D image. Merge suspended solids groups of T50, M45 and B05 seen clearly. From the 2D observation, $7.66 \times 10^{5}$ and $8.18 \times 10^{4} \mu \mathrm{m}^{2}$ of M45 and T50 have invaded from the overall porous area, $1.75 \times 10^{7}$ $\mu \mathrm{m}^{2}$, clearly about $4.84 \%$ invasion by both M45 and T50. From 3D perspective, even though the invasion area is seemingly smaller, the actual thickness of this solid film layer is relatively dense which is above quarter from the overall pore thickness. Thus, clear understanding on solid attachment and findings on permeability impairment could be directly obtained simultaneously. 


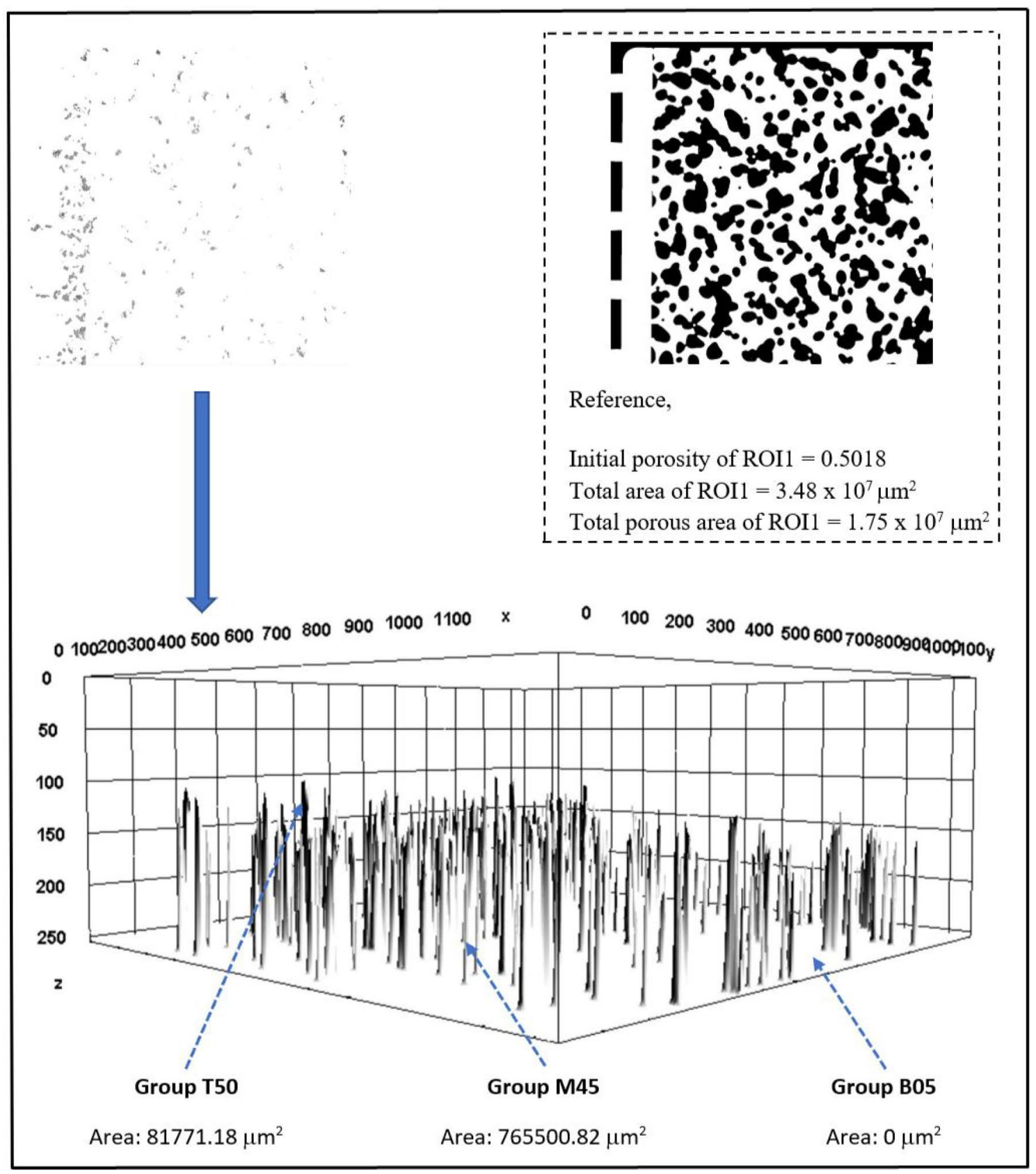

Fig. $112 \mathrm{D}$ to $3 \mathrm{D}$ visualization

\section{Conclusion and recommendation}

In a nutshell, this research study has provided an enhancement for the formation damage scope in enhanced oil recovery which is the detailed quantification method of suspended solids in porous media (micromodel) utilizing an image analysis. Also, it provides the basic knowledge on image analysis that can be implemented to maximize the executed information from the analysis and minimize the magnitude or error. On the other hand, the research output has successfully quantified the suspended solids being attached at specified 6 ROIs. First, finding indicates that there is high accumulation of suspended solids area at the inlet part (ROI1 and ROI4) compared to outlet (ROI3 and ROI6) which is due to site-constraint, inlet site prone to expose initially more rather than outlet. Second, output indicates high suspended solids of group M45 occurred at top site (ROI, ROI2, ROI3) while T50 at bottom part (ROI4, ROI5, ROI6) which is due to topological structure of the physical rock that might affect the path flow and well connectivity. Third, suspended solids 
under group M45 indicate the highest scattering index and suspension area in comparison with T50 and B05. This is because their suspension volume/ mass is relatively well dispersed which makes them invade large space area in a nick of time. Hence, through 2D porous media visualization using micromodel (image analysis) has gain firm understanding on suspended solids in pore scale. A limitation of image analysis is that it often required assumption to be made and only provides measurements of relative changes to the object of interest. An improvement that can be implemented to improve the current $2 \mathrm{D}$ pore scale visualization study is by generating the $3 \mathrm{D}$ performance using computation fluid dynamic (CFD) simulation tool, whereby it can produce quantitative predictions of fluid flow phenomena in relation with generation of suspended solids.

Supplementary Information The online version contains supplementary material available at https://doi.org/10.1007/s13202-021-01153-x.

Acknowledgments The author would like to thank Mr. Muhammad Luqman Norrani for his assistance in the laboratory.

Author Contributions Ahmad Fadhil Jahari led and designed the experimental work and data analysis. Muhammad Luqman Norrani executed the output image from micromodel test. The manuscript was written by Ahmad Fadhil Jahari. The results and manuscripts were reviewed by Siti Rohaida Mohd Shafian, Hazlina Husin, NorZafirah Razali and Sonny Irawan.

Funding This research is supported by Universiti Teknologi PETRONAS under cost center 0153CB-017.

\section{Declarations}

Conflicts of interest The authors declare no conflict of interest.

Open Access This article is licensed under a Creative Commons Attribution 4.0 International License, which permits use, sharing, adaptation, distribution and reproduction in any medium or format, as long as you give appropriate credit to the original author(s) and the source, provide a link to the Creative Commons licence, and indicate if changes were made. The images or other third party material in this article are included in the article's Creative Commons licence, unless indicated otherwise in a credit line to the material. If material is not included in the article's Creative Commons licence and your intended use is not permitted by statutory regulation or exceeds the permitted use, you will need to obtain permission directly from the copyright holder. To view a copy of this licence, visit http://creativecommons.org/licenses/by/4.0/.

\section{References}

Adrian Parsegian V (2005) Van der Waals forces: A handbook for biologists, chemists, engineers, and physicists. A Handbook for Biologists, Chemists, Engineers, and Physicists, In Van Der Waals Forces. https://doi.org/10.1017/CBO9780511614606

Barbosa, Y. M., da Silva, J. A. M., Junior, S. de O., \& Torres, E. A. (2019). Deep seawater as efficiency improver for cogeneration plants of petroleum production units. Energy, 177, 29-43. https://doi.org/ 10.1016/j.energy.2019.04.028
Belati A, Cajaiba J (2018) Measurement of wax appearance temperature using RGB image analysis and FBRM. Fuel 220:264-269. https:// doi.org/10.1016/j.fuel.2018.01.110

Chang C, Zhou Q, Kneafsey TJ, Oostrom M, Ju Y (2019) Coupled supercritical CO 2 dissolution and water flow in pore-scale micromodels. Adv Water Resour 123:54-69. https://doi.org/10.1016/j.advwatres. 2018.11.004

Craig J, Hakhoo N, Bhat GM, Hafiz M, Khan MR, Misra R, Khullar S (2018) Petroleum systems and hydrocarbon potential of the NorthWest Himalaya of India and Pakistan. Earth Sci Rev 187:109-185. https://doi.org/10.1016/j.earscirev.2018.09.012

Hernández ID, Hernández-Fontes JV, Vitola MA, Silva MC, Esperança PTT (2018) Water elevation measurements using binary image analysis for 2D hydrodynamic experiments. Ocean Eng 157(March):325-338. https://doi.org/10.1016/j.oceaneng.2018.03. 063

Khorshidian H, James LA, Butt SD (2018) Demonstrating the effect of hydraulic continuity of the wetting phase on the performance of pore network micromodels during gas assisted gravity drainage. J Pet Sci Eng 165:375-387. https://doi.org/10.1016/j.petrol.2017.11.016

Liu Y, Zhou X, You Z, Yao S, Gong F, Wang H (2017) Discrete element modeling of realistic particle shapes in stone-based mixtures through MATLAB-based imaging process. Constr Build Mater 143:169178. https://doi.org/10.1016/j.conbuildmat.2017.03.037

Mahmoodi M, James LA, Johansen T (2018) Automated advanced image processing for micromodel flow experiments; an application using labVIEW. J Petrol Sci Eng 167(January):829-843. https://doi.org/ 10.1016/j.petrol.2018.02.031

Moya AA (2015) Theory of the formation of the electric double layer at the ion exchange membrane-solution interface. Phys Chem Chem Phys 17(7):5207-5218. https://doi.org/10.1039/c4cp05702c

Paden, K (2016) Sweet Chemistry: A Study of the Intermolecular Forces in Candy Dye Molecules. Aletheia https://doi.org/10.21081/ax0015

Pham NA, Morrison A, Schwock J, Aviel-Ronen S, Lakovlev V, Tsao MS, Hedley DW (2007) Quantitative image analysis of immunohistochemical stains using a CMYK color model. Diagn Pathol 2(1):8. https://doi.org/10.1186/1746-1596-2-8

Rohaida, S., Shafian, M., Saaid, I. M., Razali, N., Jahari, A. F., Shah, M., Salleh, I. K. (2019). Aggregation of Partially Hydrophilic Silica Nanoparticles in Porous Media : Quantitative and Qualitative Analysis. Energies, 1-18.

Sharifipour M, Nakhaee A, Pourafshary P (2019) Model development of permeability impairment due to clay swelling in porous media using micromodels. J Pet Sci Eng 175:728-742. https://doi.org/10. 1016/j.petrol.2018.12.082

Shen X, Maa JPY (2016) A camera and image processing system for floc size distributions of suspended particles. Mar Geol 376:132-146. https://doi.org/10.1016/j.margeo.2016.03.009

Su P, Liang J, Peng J, Zhang W, Xu J (2018) Petroleum systems modeling on gas hydrate of the first experimental exploitation region in the Shenhu area, northern South China sea. J Asian Earth Sci 168(August):57-76. https://doi.org/10.1016/j.jseaes.2018.08.001

Xiao J, Zou C, Liu M, Zhang G, Delaplace G, Jeantet R, Chen XD (2018) Mixing in a soft-elastic reactor (SER) characterized using an RGB based image analysis method. Chem Eng Sci 181:272-285. https:// doi.org/10.1016/j.ces.2018.02.019

Yang W (2019) Finite element model of concrete material based on CT image processing technology. J Vis Commun Image Represent 64:102631. https://doi.org/10.1016/j.jvcir.2019.102631

Yu F, Jiang H, Xu F, Fan Z, Su H, Li J (2019) New insights into flow physics in the EOR process based on 2.5D reservoir micromodels. J Pet Sci Eng 181:106214. https://doi.org/10.1016/j.petrol.2019. 106214

Yuan B, Wood DA (2018) A comprehensive review of formation damage during enhanced oil recovery. J Petrol Sci Eng 167(April):287-299. https://doi.org/10.1016/j.petrol.2018.04.018

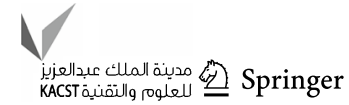


Zhang L, Xie L, Cui X, Chen J, Zeng H (2019a) Intermolecular and surface forces at solid/oil/water/gas interfaces in petroleum production. J Colloid Interface Sci 537:505-519. https://doi.org/10.1016/j. jcis.2018.11.052

Zhang Y, Zhang M, Mei H, Zeng F (2019b) Study on salt precipitation induced by formation brine flow and its effect on a high-salinity tight gas reservoir. J Petrol Sci Eng 183(June):106384. https://doi.org/10. 1016/j.petrol.2019.106384
Publisher's Note Springer Nature remains neutral with regard to jurisdictional claims in published maps and institutional affiliations. 